$\xi_{p}$

\title{
Numerical Study of Flow Characteristics Around Confined Cylinder using OpenFOAM
}

\author{
P. Mathupriya ${ }^{1}$, L. Chan ${ }^{2 *}$, H. Hasini ${ }^{3}$, A. Ooi ${ }^{4}$ \\ ${ }^{1}$ Department of Mechanical Engineering, Universiti Tenaga Nasional, Kajang 43000, Malaysia \\ ${ }^{2}$ Department of Mechanical Engineering, University of Melbourne, Victoria 3010, Australia \\ *Corresponding author E-mail : chanleonzen@gmail.com
}

\begin{abstract}
The numerical study of the flow over a two-dimensional cylinder which is symmetrically confined in a plane channel is presented to study the characteristics of vortex shedding. The numerical model has been established using direct numerical simulation (DNS) based on the open source computational fluid dynamics (CFD) code named OpenFOAM. In the present study, the flow fields have been computed at blockage ratio, $\beta$ of 0.5 and at Reynolds number, $R e$ of 200 and 300. Two-dimensional simulations investigated on the effects of Reynolds number based on the vortex formation and shedding frequency. It was observed that the presence of two distinct shedding frequencies appear at higher Reynolds number due to the confinement effects where there is strong interactions between boundary layer, shear layer and the wake of the cylinder. The range of simulations conducted here has shown to produce results consistent with that available in the open literature. Therefore, OpenFOAM is found to be able to accurately capture the complex physics of the flow.
\end{abstract}

Keywords: Blockage ratio; Confined cylinder flow; DNS; OpenFOAM; Reynolds number

\section{Introduction}

A body that is subjected to a fluid flow can be classified as a streamline or a bluff body (obstacle). A bluff body is characterized as a shape that separates the flow over substantial part of its surface. In the open literature, many researchers have shown interest in the numerical study of flow around the bluff bodies where this flow is an idealization of several industrially that used to improve the mixing and heat transfer. For an example, the cross-flow configuration in heat exchanger is observed with this configuration when the hot or cold fluid is carried by the bundle of tube, flows in perpendicular to another fluid that passes around tubes and heat is transferred between the fluids. Other applications are used with similar flow configuration such as cooling tower, electronic cooling equipment and offshore structures. The shape of the bluff body influences the formation of pressure drag. The form drag creates an additional lost that is produced when fluids is not flowing parallel to the surface This causes the change of flow direction over an obstacles. Researcher has focused on the physics of the flow like vortex shedding, and separation of flow [1]. Based on the studies, there are many difficulties in understanding the fluidstructure interaction mechanism in fundamental of physics which can be observed on the vortex shedding that causes the oscillation of the structures in the flow [2]. The vortex shedding is a significant phenomenon that can be seen at the rear of these obstacles at high Reynolds number. The vortex is defined as periodic detachment pairs of vortices where the obstacles are immersed in the flow, and it generates oscillation when the flow across the bluff body. The oscillation effects depend on the size and shape of the obstacles. Therefore, vibration effect from the oscillation plays an important role in designing the structures based on the frequency of vortex shedding and vortex analysis. As the obstacle is profiled, the velocity wake influence on the bluff body is developed from the boundary layers and wake region.

The shape such as square, triangle and circular cylinder are the examples of bluff bodies. The flow across the square cylinder in the channel is one of the significant problems in computational fluid dynamics (CFD) among other geometries. This simple geometry gives an advantage compared to other geometries because the separation is fixed at the corner and the transition of laminarturbulent will take place. The effect of the blockage ratio plays an important role in the channel flow. It influences the Strouhal number and the force coefficient. According to Turki et al, the Strouhal number and drag coefficient will increase if the blockage ratio is increased [3]. This geometry benefits the stability on transversal posture to flow. Meanwhile, the triangular shape is asymmetrical shape where it has sharp-crested face at the top and broad-crested at bottom which could cause complex laminar or turbulent flow. Recently, an incompressible flow with a triangular bluff body is investigated by Shademani et al. with a fixed blockage ratio $(\beta=0.05)$ [4]. In this work, the Reynolds number is varied and the overall framework is solved using finite volume method. The drag coefficient of the triangle decreases as the Reynolds number increases, and it gives a good agreement compared to other literature $[4,5]$.

The flow over a confined circular cylinder becomes a fundamental interest among the researchers. It creates a large drag due to the periodic separation and causes some differences in the pressure between the downstream and upstream. The dynamics of the wake flow over confined obstacles tends to be a significant effect on the heat and mass transfer to improve the thermal performance. The downstream structures of the obstacle is affected by the presence of the lateral wall in the flow that leads to an interesting fluid phenomena such as reverse von Kármán vortex street and double beating patterns in the lift coefficient of the obstacles [6]. The flow around the cylinder has been studied in three-dimensions 
(3D) by using various parameters such as blockage ratio, aspect ratio, and Reynolds number. Three-dimensional flow over a confined cylinder have been studied by Ribeiro et al. through experimental and numerical simulation using different parameters which are aspect ratio $(L / D)$ and Reynolds number [7]. Sahin et al. has focused on the flow of a confined circular cylinder with blockage ratio of $0.1<\beta<0.9$ and Reynolds number up to 280 [8]. This study reported the wall proximity effects on stability, Strouhal number, hydrodynamic forces and wake structure at the rear of the cylinder. Besides, Kanaris et al. has investigated on the three dimensional flow of confined cylinder based on the Reynolds number $(R e=10$ to 290) and blockage ratio of 0.2 [9]. The aim of the study is to investigate the force coefficients and Strouhal number. According to their studies, the grid resolution is found to influence the characteristics of the flow so careful consideration need to be made to optimize the computational cost and accuracy. The circular cylinder is chosen in this research due to the fast wake development and stronger periodic vortex shedding compared to the others [10]. There are limited literatures on the flow over a confined circular compared with unconfined circular cylinder.

There are vast of experimental studies conducted on the flow over bluff bodies for many years. However, experimental research has a certain drawbacks where it is costly and time-consuming especially for the large-scale systematic mechanism [11]. On the contrary, computational fluid dynamics (CFD) has been widely used to solve and analyze issues that involve fluid flows based on the Navier-Stokes (N-S) equations. In regards to this, the turbulence modeling plays an important role in CFD simulations where the model is used to forecast the turbulence effects. This turbulence appeared dominant over the other flow phenomena whenever it occurs in a particular flow which causes the turbulence modeling to enhance the quality of numerical simulations. There are few methods which are used to solve the NavierStokes equations. The methods are direct numerical simulation (DNS), large eddy simulation (LES) and Reynolds-averaged Navier-Stokes (RANS). Both LES and RANS requires a turbulence model to approximate the effects of turbulence whereas DNS solves the N-S directly.

However, LES is not compatible for small-scale turbulent flow as it is unable to trap the vortices around the surface of the cylinder. Besides, RANS is able to simulate the large turbulent eddies in the flow whereas the remaining eddies are modelled. This allows for a coarse grid to be used. Although RANS was applied for smallscale flow, the result obtained is not accurate as compared with DNS. It is also could unable to predict any separation occurred in turbulence model [12]. DNS gives an accurate solution compared with other methods by resolving turbulent motion with a fine mesh in order of Kolmogorov microscales. Based on the studies conducted by Kim et al, the first DNS performed on fully developed turbulent channel flow with $4 \times 10^{6}$ grid-mesh and Reynolds number of 3300 based on centreline velocity and channel halfwidth which this equates to a friction at Reynolds number of 180 [13]. Since then, with the advancement in computational power, more complex flows has been simulated at higher Reynolds number to have a better understanding of the transport mechanism. DNS used in flow over obstacles to identify the characteristics of turbulent features in a von Kármán vortex street. For instance, the flow of cylinder at $R e=3900$ is investigated by MA et al. using DNS [14]. Based on the results, the mean velocity profile at the vicinity of the cylinder showed a good agreement with experimental data. This method has been used by Jiang et al. [15] to discover large-scale vortex dislocations (Mode A) for the flow over a circular cylinder in three-dimensional. The author stated that high accuracy schemes such as spectral element method can be obtained through DNS. Therefore, DNS is a useful simulation to analyze the physics of turbulence [16] and able to extract data easily.

In the present paper, the potential of the open-source CFD software tool OpenFOAM (Open Field Operation and Manipulation) is used to simulate the flow around a confined cylinder. The current two-dimensional simulation blocked channel is studied with different numerical solvers from OpenFOAM. DNS method is used in this study as it is a powerful tool to study any complex flow phenomenon and it resolves all the turbulence scales. Twodimensional simulation is carried out with blockage ratio, $\beta$ and various Reynolds number, $R e$. The unsteady flow is analyzed to identify the flow characteristics based on the force coefficients and Strouhal number.

\section{Computational Setup}

The mesh structure for the flow around a confined cylinder in this study is a rectangular domain with hexahedron cells (prism with quadrilateral base). The cylinder is placed symmetrically with fixed wall boundary condition at the top and bottom of the lateral walls, where the height of lateral wall $-1<H<1$ (wall-normal direction), length of the domain is placed at the distance of $\mathrm{L}_{1}=$ 10 upstream of the cylinder and the outlet is located at $\mathrm{L}_{2}=30$ downstream of the cylinder shown in figure 1 . The construction of the confined cylinder is based on the blockage ratio $\beta=D / H=0.5$ where $D$ is diameter of the cylinder. $U$ is the centreline inflow velocity.

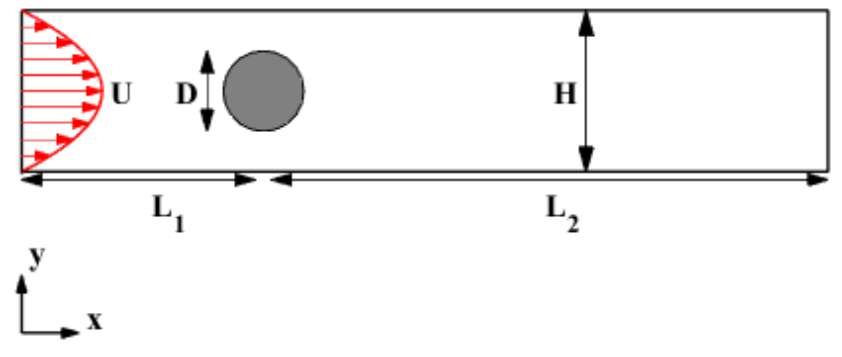

Figure 1: The sketches of the computational domain for a confined cylinder in the channel.

The diameter of the cylinder is 1 , and it assumed as a fixed confined cylinder that will not move even if subjected to forces. The center is located at $(0,0)$ whereas the mesh consists of 83 vertices and 27 blocks at front and back of the domain. Figure 2 shows computational mesh of the domain expanded view in the vicinity of the cylinder.

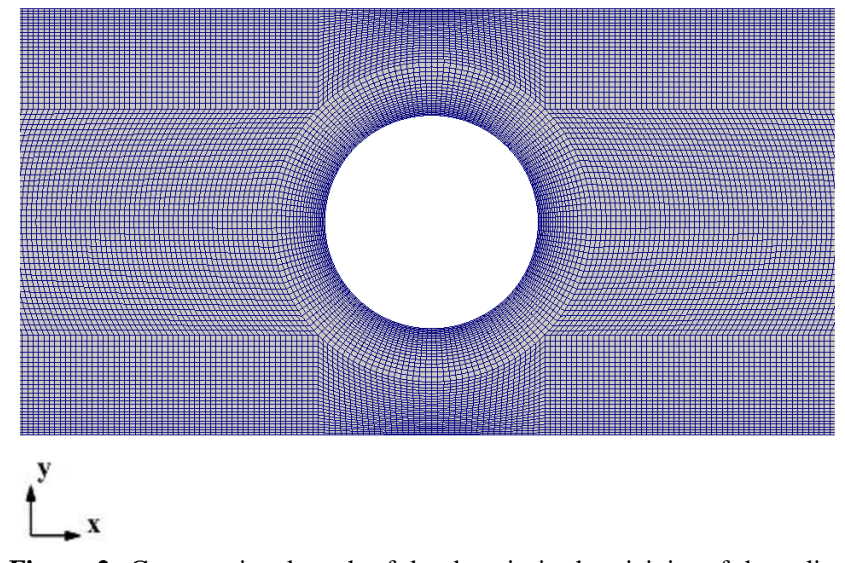

Figure 2: Computational mesh of the domain in the vicinity of the cylinder.

The computational mesh is changed accordingly to the blockage ratio, and the size of the mesh around the cylinder is refined to obtain an accurate result for the flow simulation. The refinement is modified through grading scale under the blocks section through 'blockMeshDict'file. Three types of meshes are tested namely, case 1 - coarse mesh, case 2 - medium mesh, and case 3 - fine mesh to observe the simulation from this grid. The meshes of each 
case are characterized by $\mathrm{N}_{\text {cyl }}$ : Number of nodes at the surface of the cylinder, $\mathrm{N}_{\max }$ : Number of nodes from cylinder to the outflow boundary and $\mathrm{N}_{\text {wall }}$ : Number of nodes between the cylinder and wall as shown in table 1 . The coarse and fine mesh are considered based on the $20 \%$ of the number of nodes at the cylinder surface from the medium mesh.

Table 1: Values of grid parameters for a confined cylinder with $\beta=0.5$. Case 1 has a coarse mesh, case 2 a medium mesh and case 3 a fine mesh.

\begin{tabular}{|l|c|c|c|c|}
\hline Cases & $\mathbf{N}_{\text {cyl }}$ & $\mathbf{N}_{\max }$ & $\mathbf{N}_{\text {wall }}$ & $\mathbf{N}_{\text {Cells }}$ \\
\hline Case 1 & 256 & 2070 & 82 & 431896 \\
\hline Case 2 & 328 & 2587 & 102 & 620792 \\
\hline Case 3 & 392 & 3104 & 122 & 893158 \\
\hline
\end{tabular}

In this study, the force of fluids is calculated and gained a better understanding on the impact of fluids passing through an object The Navier-Stokes equations are governed by equations for continuum medium which is a viscous fluid. The incompressible unsteady Navier-Stokes equations can be written in the following form:

$\nabla \cdot \mathbf{u}=0$

$\frac{\partial \mathbf{u}}{\partial t}+(\mathbf{u} \cdot \nabla) \mathbf{u}=-\nabla p+\frac{1}{\operatorname{Re}} \nabla^{2} \mathbf{u}$

Where $\mathbf{u}$ is the velocity vector, $p$ represents the pressure and $R e$ is the Reynolds number, given by:

$R e=\frac{U_{\max } D}{v}$

where $U_{\max }$ is the maximum inlet velocity of the fluid and $v$ is the kinematic viscosity. The nodal-based finite-volume code is used to perform the current computations. The second-order centering differencing scheme is used for spatial discretization. The gauss linearupwindV will be used for other spatial term and the secondorder accurate backward scheme is used for time discretization. The inlet of the domain for velocity follows the parabolic profile, where

$U_{x}=\left[1-y^{2}\right]$

is the velocity profile set for the channel simulations and $y$ is the height of the channel in wall-normal direction. No-slip boundary condition imposed on the cylinder in a channel. A zero-gradient boundary condition is applied to the outlet so that the flow structure convects out of the computational domain without being distort. The maximum inlet velocity is set to be 1 . The values of Reynolds numbers, $R e$ used in this study are 200 and 300.

\section{Solution Setup}

\subsection{Input / Output Control}

In OpenFOAM solvers, it is able to set up data that controls input/output through 'controlDict' which creates database of time step and time interval. The Courant number is required to be sufficiently small to ensure stability and accuracy of simulation. For all of the cases, a Courant number of less than 0.2 is used. The force and force coefficient are added in 'controlDict' which it will be automatically extracted the value of lift and drag coefficient from the simulation.

\subsection{Solvers}

Three types of transient solver are used for incompressible flow which are icoFoam, pisoFoam, and pimpleFoam. icoFoam is a solver which solves the turbulent flow of Newtonian fluids based on the Navier-Stokes. This solver will be used to solve case 1, 2 and 3. The pimpleFoam is a solver for incompressible flow based on large time-step by using PIMPLE (merged of PISO-SIMPLE) algorithm whereas pisoFoam is a transient solver for incompressible flow that runs with time step. Both pimpleFoam and pisoFoam are used to solve medium mesh (case 2).

\subsection{Post-Processing}

The simulations are performed over a period of approximately 250 dimensionless time units when the flow has reached fully converged state. The results obtained from the simulation are pressure, velocity and force coefficients according to time histories. The results of force coefficient including lift and drag coefficients on the confined cylinder flow can be extracted from 'forceCoeffs' file. Some important informations such as the diameter of the cylinder, reference area, and reference length should be provided to generate accurate force coefficients. There are two types of forces acted on the confined cylinder during inflow which are drag force and lift force. These forces are identified by integrating the shear stress and pressure along the surface of the cylinder. The dimensionless drag and lift coefficient are defined as:-

$$
\begin{gathered}
C_{L}=\frac{F_{L}}{0.5 U_{\text {max }}^{2} D} \\
C_{D}=\frac{F_{D}}{0.5 U_{\text {max }}^{2} D}
\end{gathered}
$$

where $F_{L}$ is lift force, and $F_{D}$ is drag force. Generally, the simulations are able to measure the frequency of vortex shedding. The vortex shedding is identified based on Strouhal number which is defined as:

$$
S t=\frac{D f}{U_{\max }}
$$

where $f$ is the period of vortex shedding which the results obtained are determined based on the time resolved lift cofficient through the spectral analysis.

\section{Validation of Numerical Code}

\subsection{Grid Independence Study}

The study of grid independence is required to ensure that the solution is independent to the size of the grid. Mesh of these densities, coarse, medium, and, fine are created and tested in this study. These simulations are conducted using icoFoam solver. From the results of the simulation, $\overline{\mathbf{C}_{\mathbf{D}}}$ and $\overline{\mathbf{C}_{\mathbf{L}}}$ which are the timeaveraged of drag and lift coefficients calculated as shown in table 2 . The mean value is obtained from the time taken by the periodic behavior of lift coefficient in a steady state. The Strouhal number, $S t$ is calculated to identify the oscillating flow mechanisms based on the frequency of vortex shedding.

Table 2: Results of the grid dependency study for $\beta=0.5$ and $R e=200$. Case 1 has a coarse mesh, case 2 a medium mesh and case 3 a fine mesh.

\begin{tabular}{|c|c|c|c|}
\hline Case & $\overline{\mathbf{C}_{\mathbf{D}}}$ & $\overline{\mathbf{C}_{\mathbf{L}}}$ & $\mathbf{S t}$ \\
\hline $\mathbf{1}$ & 2.4217 & 0.0012 & 0.3815 \\
\hline $\mathbf{2}$ & 2.4217 & $5.74 \mathrm{E}-05$ & 0.3815 \\
\hline $\mathbf{3}$ & 2.4217 & $6.02 \mathrm{E}-05$ & 0.3815 \\
\hline
\end{tabular}




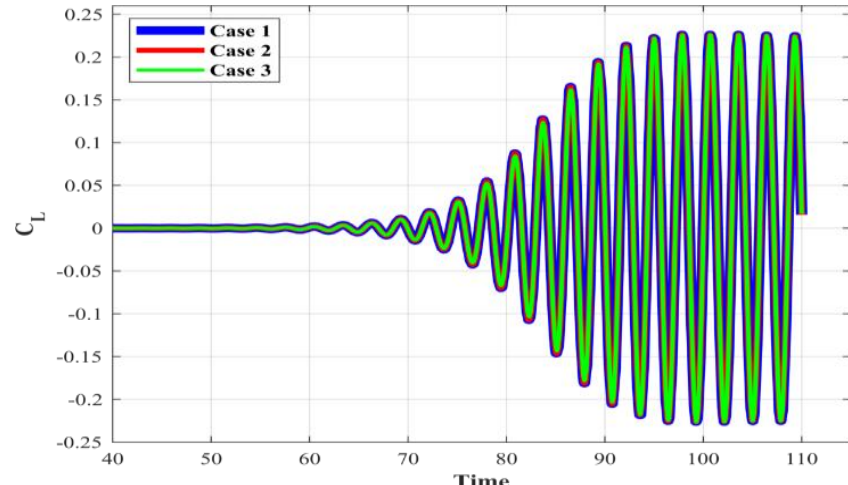

Figure 3: Lift coefficient for case 1, 2 and 3. The case 1 represented in blue, case 2 represented in red and green for case 3 .

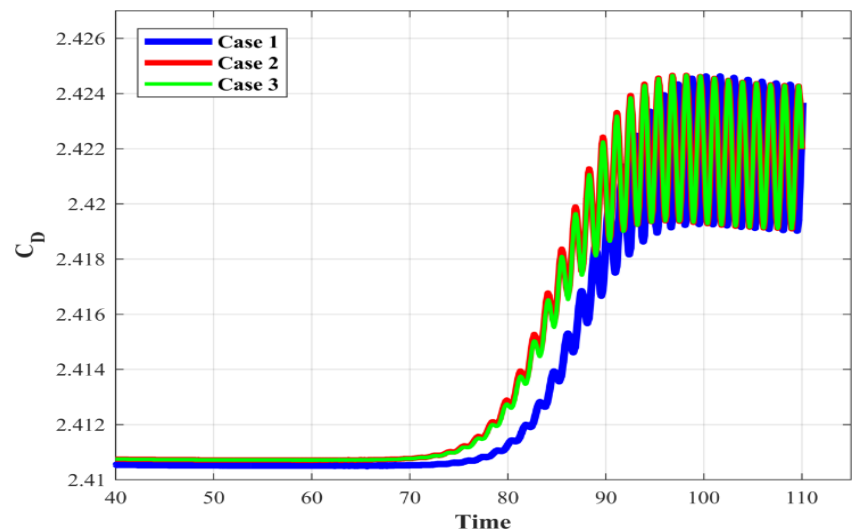

Figure 4: Drag coefficient for case 1, 2 and 3. The case 1 represented in blue, case 2 represented in red and green for case 3 .

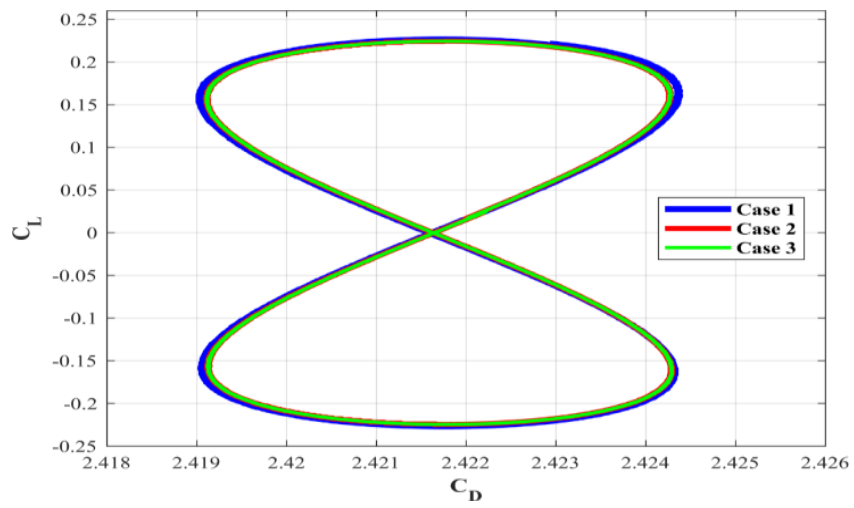

Figure 5: Lift and drag coefficients of case 1, 2 and 3. The case 1 represented in blue, case 2 represented in red and green for case 3.

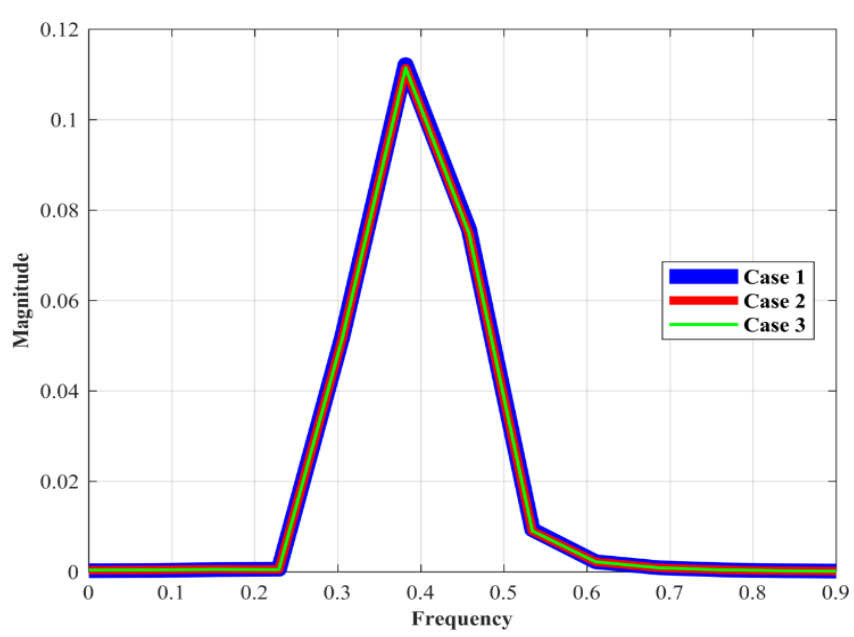

Figure 6: The spectral analysis of case 1,2 and 3. The case 1 represented in blue, case 2 represented in red and green for case 3 . This plot indicates the frequency of Strouhal number.
The profiles of time and force coefficient for case 1, 2 and 3 with blockage ratio, $\beta=0.5$ are shown in figure 3,4 , and 5 respectively. The frequency of Strouhal number of three cases shown in figure 6 are found to be similar. This analysis showed a good agreement with each other, and it showed that this simulation is consistent although the number of cells in each case are varied. Thus, case 2 is chosen for further unsteady flow simulation where else in case 3 , longer computational time is observed. The time-averaged lift coefficient, $\mathrm{C}_{\mathrm{L}}$ should be zero value which is similar in case 2 .

\subsection{Validation of Different Numerical Solver}

Another validation is carried out where the simulations of case 2 based on three transient solvers which are icoFoam, pimpleFoam, and pisoFoam. The results obtained from this analysis is compared with [8] to validate the accuracy of the numerical code. The results of time-dependent force coefficient are presented in figures 7,8 and 9 for $\beta=0.5$ at $\operatorname{Re}=200$.

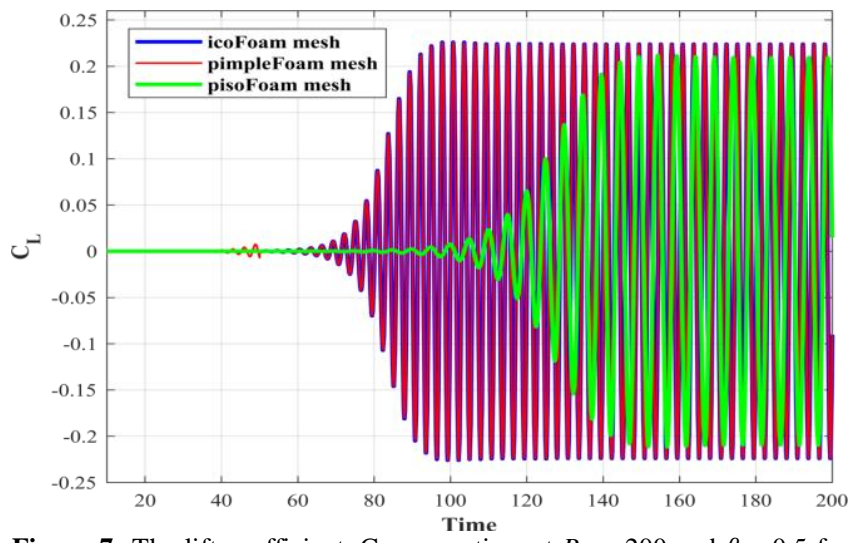

Figure 7: The lift coefficient, $\mathrm{C}_{\mathrm{L}}$ versus time at $R e=200$ and $\beta=0.5$ for case 2 using icoFoam, pimpleFoam, and pisoFoam. The icoFoam represented in blue, pimpleFoam represented in red and green for pisoFoam.

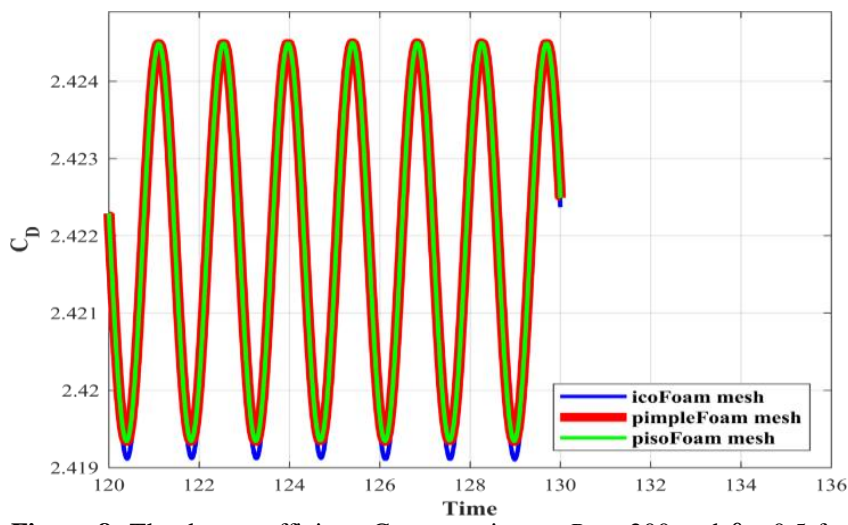

Figure 8: The drag coefficient, $\mathrm{C}_{\mathrm{d}}$ versus time at $R e=200$ and $\beta=0.5$ for case 2 using icoFoam, pimpleFoam, and pisoFoam. The icoFoam represented in blue, pimpleFoam represented in red and green for pisoFoam.

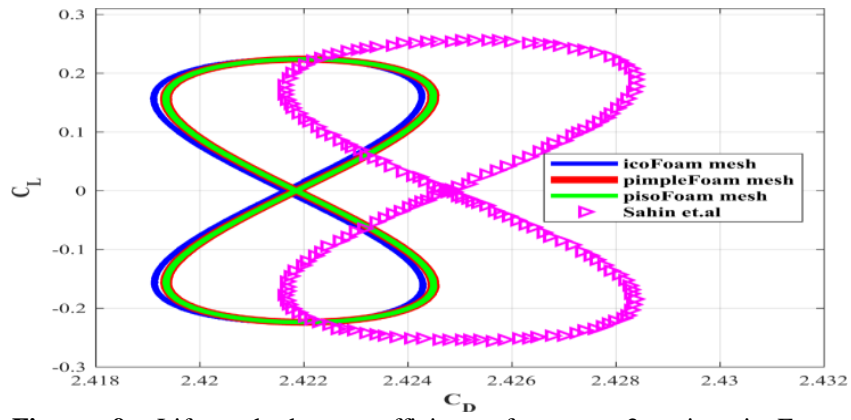

Figure 9: Lift and drag coefficients for case 2 using icoFoam, pimpleFoam, and pisoFoam. The icoFoam represented in blue, pimpleFoam represented in red, green for pisoFoam and Sahin et al. [8] indicated in purple and right-pointing triangle of marker type. 


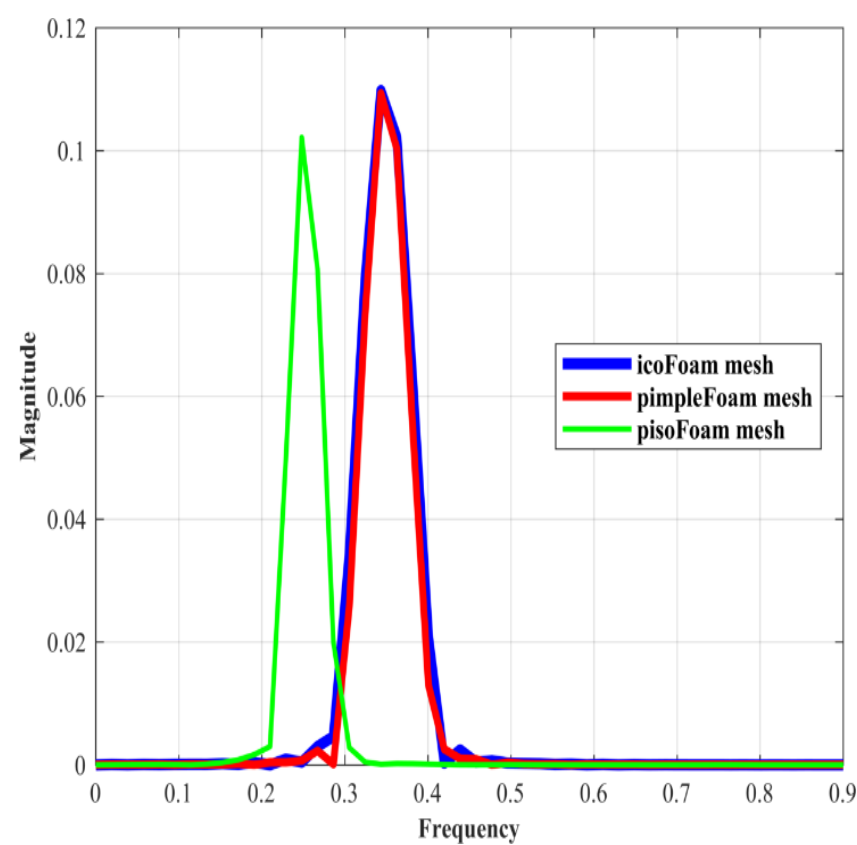

Figure 10: The spectral analysis for case 2 using icoFoam, pimpleFoam, and pisoFoam. The icoFoam represented in blue, pimpleFoam represented in red, green for pisoFoam. This plot indicates the frequency of Strouhal number.

Table 3: Results of the various numerical solver. This table indicated with three type of solver namely, icoFoam, pimpleFoam and pisoFoam. These solvers are compared with Sahin et.al [8].

\begin{tabular}{|c|c|c|c|}
\hline Solver & $\overline{\mathbf{C}_{\mathbf{D}}}$ & $\overline{\mathbf{C}_{\mathbf{L}}}$ & $\mathbf{S t}$ \\
\hline icoFoam & 2.4217 & $5.74 \mathrm{E}-05$ & 0.3433 \\
\hline pimpleFoam & 2.4219 & $-6.35 \mathrm{E}-05$ & 0.3433 \\
\hline pisoFoam & 2.4217 & 0.0012 & 0.248 \\
\hline Sahin et.al [8] & 2.4249 & -0.0035 & 0.3513 \\
\hline
\end{tabular}

Figure 7 and figure 8 show the plot of lift coefficient and drag coefficient against the time with three transient solvers. Based on this graph, the pisoFoam solver takes longer time to converge compared with other solvers. The icoFoam and pimpleFoam shows similar results where these solvers are in fully converged. The results and analyses of lift and drag coefficient of case 2 shown in figure 9 able to measure the frequency of vortex shedding. There is dominant Strouhal number appeared in flow over cylinder case for $R e=200$ with $\beta=0.5$ as shown in figure 10 . This frequency is identified through spectral analysis on the lift coefficient of the cylinder. The frequency obtained in this case is $f=$ 0.3433 where it show similar results with icoFoam and pimpleFoam. The frequency of pisoFoam shows the lowest value as it takes longer time to be fully converged. Little differences observed in the force coefficients could be noticed for the three transient solvers. The results are tabulated in table 3. Hence, icoFoam solver is chosen for the subsequent part of the analyses because it proves that this solvers is very close to zero value of time-averaged lift coefficient, $C_{L}$ compared with pimpleFoam. The results of the simulation are validated with the data of [8]. Overlaying [8] results in figure 9, it initially appears as if the current simulation results significantly underpredicts the drag coefficient of the cylinder. However, the difference in Strouhal number between the current simulation (icoFoam and pimpleFoam) and [8] are approximately $2.3 \%$ where it shows a good agreement between both studies.

\subsection{Flow Simulation at $R e=200$}

The visualization of pressure, velocity profiles and vorticity contour for the flow $\operatorname{Re}=200$ with blockage ratio of $\beta=0.5$ are shown in figure 11,12 and 13.

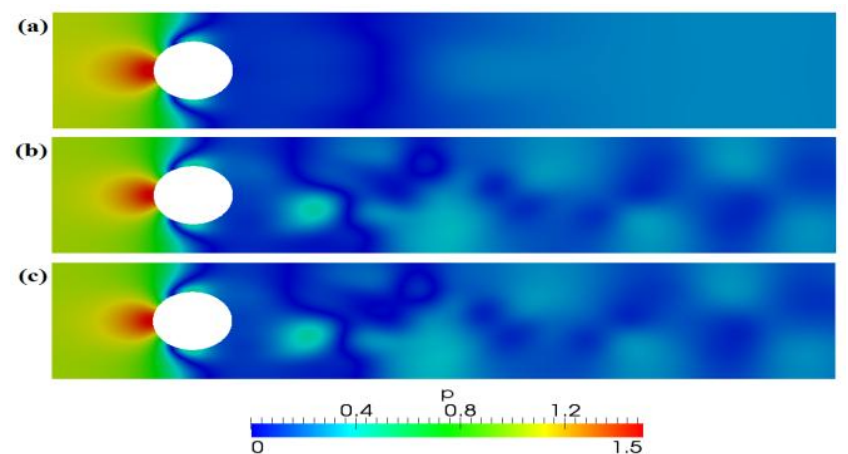

Figure 11: Pressure profile at $R e=200$ and $\beta=0.5$ at times of $t=$ (a) 50 $D / U_{\max }$, (b) $100 \mathrm{D} / U_{\max }$, and (c) $120 \mathrm{D} / U_{\max }$.

The periodic variations in the wake behind the obstacle can be seen by the pressure contours around the cylinder, as shown in three sequences of pressure field in figure 11. The figure reveals that the periodic formation of low and high pressures in the far downstream of the cylinder. The low pressures show at the behind of the cylinder and the high pressures observed at the front of stagnation region.

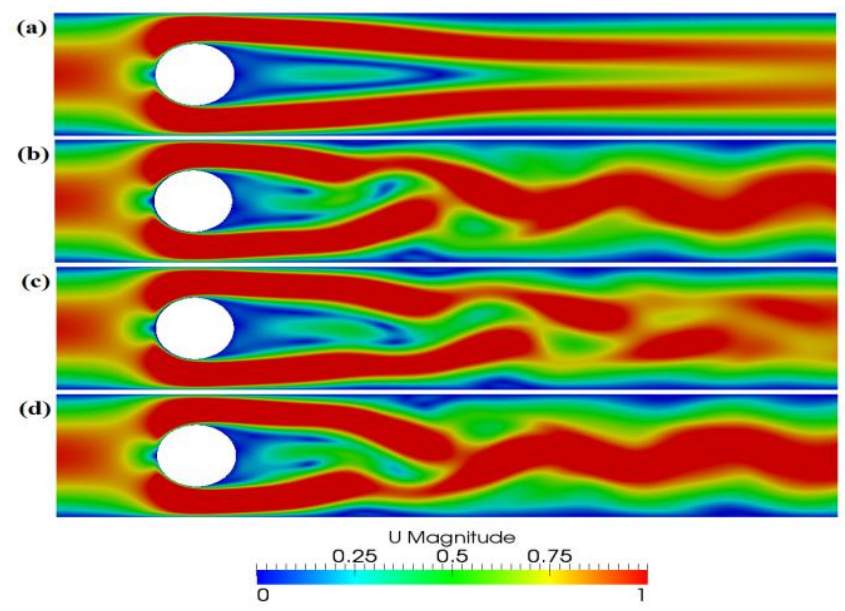

Figure 12: Velocity profile at $R e=200$ and $\beta=0.5$ at times of $t=$ (a) 50 $D / U_{\max }$, (b) $80 \mathrm{D} / U_{\max }$, (c) $90 \mathrm{D} / U_{\max }$, and (d) $100 \mathrm{D} / \mathrm{U}_{\max }$.

Figure 12 shows the velocity contours in the vicinity of the cylinder for three successive moments at the time of $t=50 \mathrm{D} / \mathrm{U}_{\max }, 80$ $D / U_{\max }, 90 \mathrm{D} / U_{\max }$, and $100 \mathrm{D} / U_{\max }$. It can be seen from the figure 12(a) - (d), vortices form behind the circular cylinder is called as vortex shedding especially. At higher $R e$, the wake becomes unsteady, and the vortices are shed from each side of the circular cylinder.

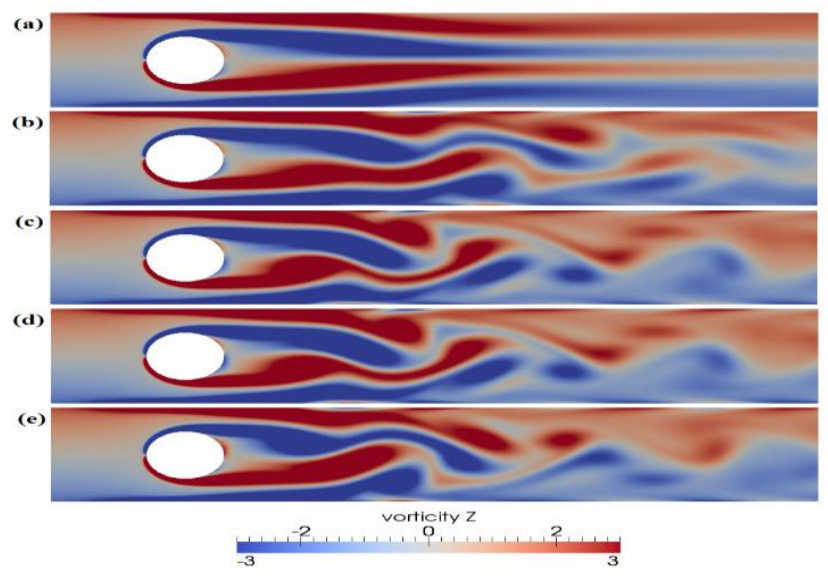

Figure 13: Spanwise vorticity contours of the periodic flow at $R e=200$ and $\beta=0.5$ at times of $t=$ (a) $50 \mathrm{D} / U_{\max }$, (b) $80 \mathrm{D} / U_{\max }$, (c) $100 \mathrm{D} / U_{\max }$, (d) $120 \mathrm{D} / \mathrm{U}_{\max }$ and (e) $150 \mathrm{D} / \mathrm{U}_{\max }$. 
The five sequences of vorticity using icoFoam solver are shown in figure 13. Vorticity occured when flows are separated from the surface of the cylinder. Shear layer is formed and causes it to change into vortex which it shed from circular cylinder. Based on the simulation result shown in figure 13(a) - (e), the vortex shedding phenomenon is created at the cylinder and the channel walls. The wake became unstable at this range, and it shows result in vortex shedding that oscillates at a certain frequency. When the vortices move downstream, the trajectories of clockwise vortices shed at the top of cylinder then cross to the bottom of the cylinder, and therefore effects of proximity can be viewed.

\subsection{Flow Simulation at $R e=300$}

The visualization of pressure, velocity profiles and vorticity contour for the flow $\operatorname{Re}=300$ with blockage ratio of $\beta=0.5$ are shown in figure 14,15 and 16 .

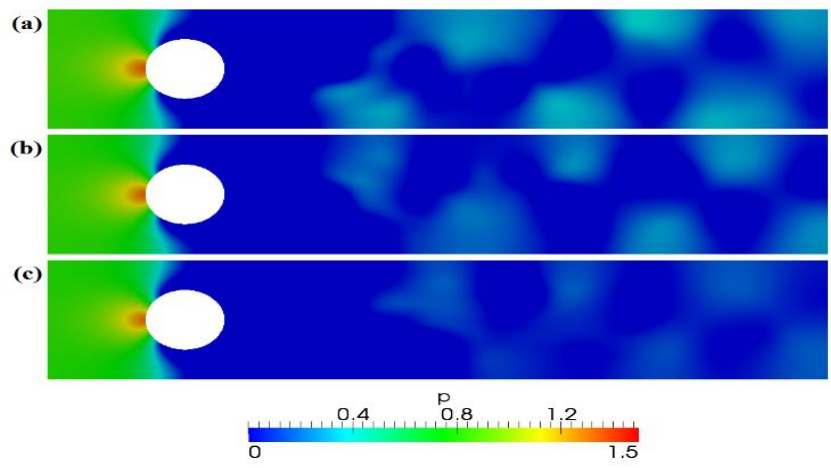

Figure 14: Pressure profile at $\operatorname{Re}=300$ and $\beta=0.5$ at times of $t=$ (a) 80 $\mathrm{D} / U_{\max }$, (b) $90 \mathrm{D} / U_{\max }$, and (c) $100 \mathrm{D} / U_{\max }$.

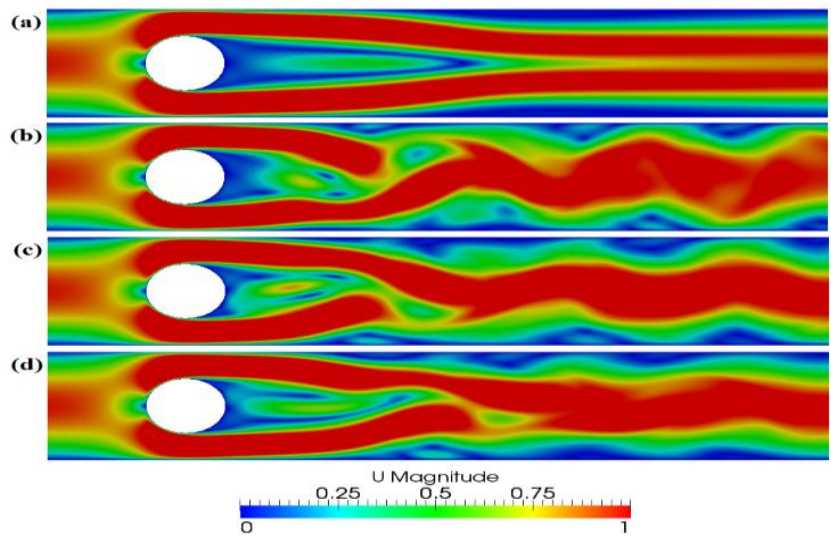

Figure 15: Velocity profile at $\operatorname{Re}=300$ and $\beta=0.5$ at times of $t=$ (a) 50 $\mathrm{D} / U_{\max }$, (b) $80 \mathrm{D} / U_{\max }$, (c) $90 \mathrm{D} / U_{\max }$ and (d) $100 \mathrm{D} / U_{\max }$

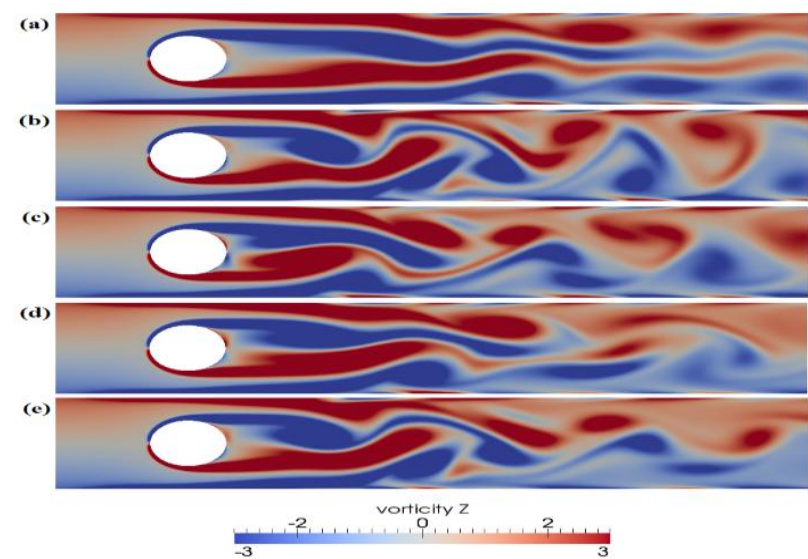

Figure 16: Spanwise vorticity contours of the periodic flow at $R e=200$ and $\beta=0.5$ at times of $t=$ (a) $70 \mathrm{D} / U_{\max }$, (b) $80 \mathrm{D} / U_{\max }$, (c) $90 \mathrm{D} / U_{\max }$, (d) $100 \mathrm{D} / U_{\max }$ and (e) $150 \mathrm{D} / U_{\max }$
Based on figures 14 and 15, the flow qualitatively exhibits the same pattern as shown in figure 11 and 12 . The vorticity contour is shown in figure 16 is separated by five periods of the $70 \mathrm{D} / \mathrm{U}_{\max }$, $80 \mathrm{D} / \mathrm{U}_{\max }, 90 \mathrm{D} / \mathrm{U}_{\max }, 100 \mathrm{D} / \mathrm{U}_{\max }$, and $150 \mathrm{D} / \mathrm{U}_{\max }$. The vortices are formed on the wall of the channel which shows that is entrained into vortex street. The separation appeared when a large vortex cut off and shed that creates small vortex which eventually become larger and separation continues. In figures 16(b) and (e), the vortices appeared to become counteractive and the period of beating looks nearly identical and quite similar to the vorticity simulations resulted in [6].

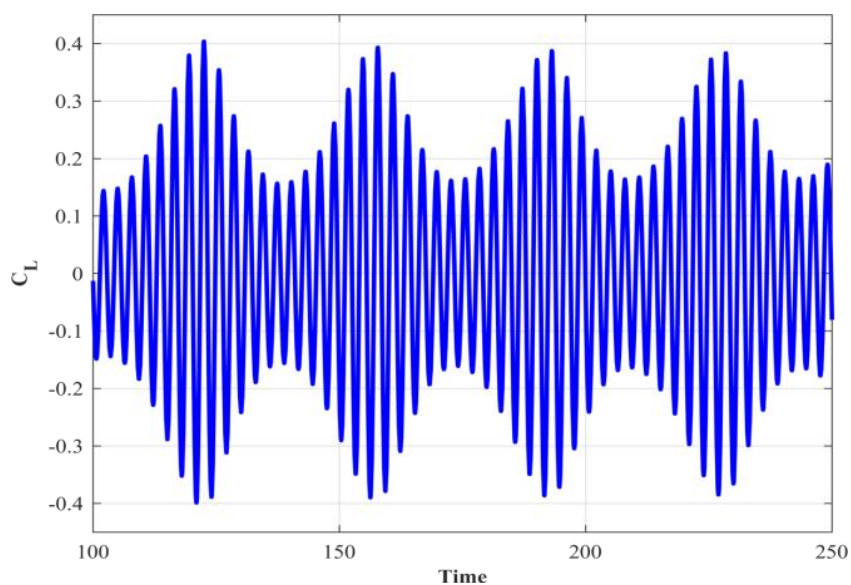

Figure 17: The lift coefficient, $\mathrm{C}_{\mathrm{L}}$ versus time at $R e=300$ and $\beta=0.5$.

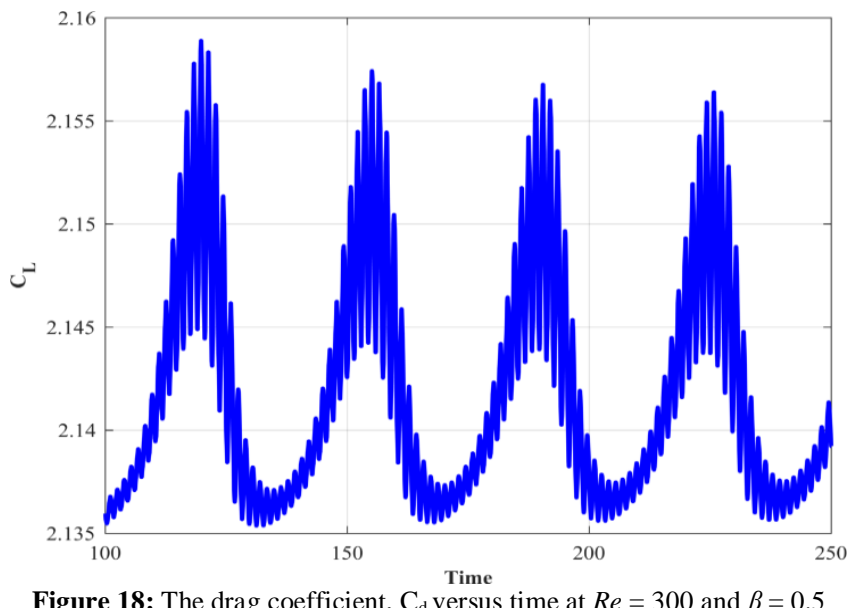

Figure 18: The drag coefficient, $\mathrm{C}_{\mathrm{d}}$ versus time at $R e=300$ and $\beta=0.5$

The lift coefficient, $C_{L}$ and drag coefficient, $C_{D}$ are plotted against with time as shown in figures 17 and 18 for $R e=300$ and $\beta=0.5$ respectively. Based on the graph, a low-frequency beating appeared where this phenomenon shows two different frequencies.

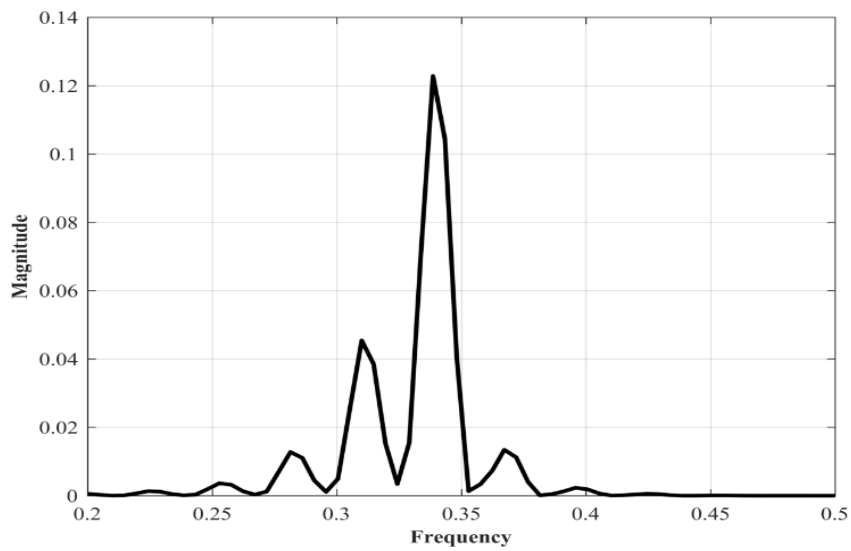

Figure 19: The spectral analysis at $\operatorname{Re}=300$ and $\beta=0.5$. This plot indicates the frequency of Strouhal number. 
The spectral analysis is plotted in figure 19 to identify the Strouhal number based on the lift coefficient. This frequency is obtained by the lift coefficient of the cylinder via spectral analysis. Based on the graph, the Strouhal number is observed at $\mathrm{St}=0.3386$ and 0.3099 . These frequencies corresponded to the double beating as shown in figure 17 and also it is noticable in drag coefficient as in figure 18. The confined wall may contribute to the double beating as the vortices apparently hit the wall at higher Reynolds number. The Strouhal number reported in [6] are 0.513 and 0.468 . The percentage error between both results are approximately $34 \%$. The reason that can be considered, which are the inlet and outlet length set used in [6] are $\mathrm{L}_{\mathrm{i}}=12 \mathrm{D}$ and $\mathrm{L}_{\mathrm{o}}=45 \mathrm{D}$ whereas in the current study, the lengths are set with $-10<\mathrm{x} / \mathrm{D}<30$ (streamwise). To further explored the phenomenon of double beating frequency, three-dimensional simulations of similar parameters must be carried out. OpenFOAM software is able to identify the changes of Strouhal number accurately in accordance to the changes of Reynolds number.

\section{Conclusion}

The flow over a circular cylinder placed symmetrically in a channel is simulated based on the finite-volume code by using direct numerical simulation (DNS). This simulation is done by using OpenFOAM which is an open source computational fluid dynamics (CFD) code. The two-dimensional simulations are carried out with a blockage ratio, $\beta=0.5$ and Reynolds number, $R e=200$ and 300 . The grid independence study is carried out to make sure that results obtained are independent to the size of the grid. Next, the validation of the numerical solver is conducted where three transient solvers which are icoFoam, pimpleFoam and pisoFoam are tested for a flow over a cylinder confined in a channel. All three solvers are found to be accurately reproduced the lift and drag coefficients and also the frequency of the shedding. The flow behavior over circular cylinder is identified based on the contours of velocity, pressure, and vorticity at $R e=200$ and 300. There are two different frequencies appeared at higher Reynolds number which can be caused by the confinement effects of the channel. The validation of the OpenFOAM solvers are found to be consistent with the published results in the literature. The solver in OpenFOAM exhibited as a compatible solver that able to capture the complex dynamics of the flow over a confined cylinder including the interactions between the shear layer from the walls and the flow separation and turbulent wake from the cylinder.

\section{Acknowledgment}

The financial assistance received from UNITEN BOLD scholarship and UNITEN BOLD internal grant is greatly appreciated.

\section{References}

[1] M. I. Yuce and D. A. Kareem, "A numerical analysis of fluid flow around circular and square cylinders," Am. Water Works Assoc., vol. 108 , p. 10,2016

[2] S. Bhadauriya, H. Kapadia, A. Dalal, and S. Sarkar, "Effect of channel confinement on wake dynamics and forced convective heat transfer past a blunt headed cylinder," Int. J. Therm. Sci., vol. 124, pp. 467-476, 2018.

[3] S. Turki, H. Abbassi, and S. B. Nasrallah, "Effect of the blockage ratio on the flow in a channel with a built-in square cylinder," Comput. Mech., vol. 33, no. 1, pp. 22-29, 2003.

[4] R. Shademani, P. Ghadimi, R. Zamanian, and A. Dashtimanesh, "Assessment of Air Flow over an Equilateral Triangular Obstacle in a horizontal Channel Using FVM,” J. Math. Sci. Appl., vol. 1, no. 1, pp. 12-16, 2013

[5] A. Dhiman and R. Shyam, "Unsteady heat transfer from an equilateral triangular cylinder in the unconfined flow regime," ISRN Mech. Eng., vol. 2011, 2011.
[6] M. D. Griffith, J. Leontini, M. C. Thompson, and K. Hourigan, "Vortex shedding and three-dimensional behaviour of flow past a cylinder confined in a channel,” J. Fluids Struct., vol. 27, no. 5, pp. 855-860, 2011.

[7] V. M. Ribeiro, P. M. Coelho, F. T. Pinho, and M. A. Alves, "Threedimensional effects in laminar flow past a confined cylinder," Chem. Eng. Sci., vol. 84, pp. 155-169, 2012.

[8] M. Sahin and R. G. Owens, "A numerical investigation of wall effects up to high blockage ratios on two-dimensional flow past a confined circular cylinder," Phys. Fluids, vol. 16, no. 5, pp. 1305 1320, 2004.

[9] N. Kanaris, D. Grigoriadis, and S. Kassinos, "Three dimensional flow around a circular cylinder confined in a plane channel," Phys. Fluids, vol. 23, no. 6, p. 064106, 2011.

[10] F. S. Pereira, G. Vaz, and L. Eça, "Flow Past a Circular Cylinder: A Comparison Between RANS and Hybrid Turbulence Models for a Low Reynolds Number," in 34th International Conference on Ocean, Offshore and Artic Engineering, 2015.

[11] H. Zheng, Y. Li, and L. Cai, "Research on performance of H2 rich blowout limit in bluff-body burner," Math. Probl. Eng., vol. 2012, 2012.

[12] R. Ranjan, S. Deshpande, and R. Narasimha, "A Critical Comparison of RANS, LES, Hybrid LES/RANS and DNS Studies of the Flow Past a Low Pressure Turbine Blade in a Cascade," 2016.

[13] J. Kim, P. Moin, and R. Moser, "Turbulence statistics in fully developed channel flow at low Reynolds number," J. Fluid Mech., vol. 177, pp. 133-166, 1987.

[14] X. MA, G.-S. Karamanos, and G. E. Karniadakis, "Dynamics and low-dimensionality of a turbulent near wake," J. Fluid Mech., vol. 410, pp. 29-65, 2000.

[15] H. Jiang, L. Cheng, F. Tong, S. Draper, and H. An, "Stable state of Mode A for flow past a circular cylinder," Phys. Fluids, vol. 28, no. 10, p. 104103, 2016.

[16] M. S. Triantafyllou, F. S. Hover, and A. H. Techet, "The effect of Reynolds number on VIV: from subcritical to supercritical flow," in Proc. 8th Intl Conf. on Flow Induced Vibrations, FIV, 2004, p. 303. 